\title{
Filter Structures Based on Basic, Dynamic Nonstationary Elements
}

\author{
Roman Kaszyński, Adrian Sztandera, Katarzyna Wiechetek \\ Department of Systems, Signals and Electronic Engineering Faculty of Electrical West Pomeranian University of Technology, Szczecin, \\ Poland
}

\section{Email address:}

roman.kaszynski@zut.edu.pl (R. Kaszyński), adrian.sztandera@gmail.com (A. Sztandera), wiechetekkatarzyna@gmail.com (K. Wiechetek)

\section{To cite this article:}

Roman Kaszyński, Adrian Sztandera, Katarzyna Wiechetek. Filter Structures Based on Basic, Dynamic Nonstationary Elements. Automation, Control and Intelligent Systems. Vol. 3, No. 6, 2015, pp. 128-132. doi: 10.11648/j.acis.20150306.16

\begin{abstract}
The results of basic dynamic elements research of filters with time-varying parameters which are low-pass filters, high-pass filters, and band-stop filters are presented. With the use of those elements more complex nonstationary structures were created. This provided the capability of forming spectral characteristics. Examples of filtration presented in this paper showed the reduction of transient state. Presented study assumes, that it is possible to create complex structures with required spectral properties.
\end{abstract}

Keywords: Transient State, Time-Varying Parameters, Spectral Properties, Basic Nonstationary Elements, Complex Structures

\section{Introduction}

Presented study assumes that the dynamic, nonstationary high-pass and low-pass first order elements are basic structures. Inertial element was modified with gain function $k(t)$ and time constant function $T(t)[6,11]$. To the high-pass element, which is an derivative real term, functions varying the gain and time in such way as it is changed in inertial element, at the same time leaving the derivative time constant were implemented. The values of time-varying parameters are settling with time approaching to infinity. After terminating the transient state with demanded accuracy it provides required spectral characteristic $[1,7,10,11]$.

The fact, that the time-varying parameters settle their values in time is important in term of stationarity of filters output signals. Then, it is possible to determine their frequency characteristics and establishing whether all assumptions are fulfilled. In the dynamic systems described by the differential equations, nonstationarity is a feature of transients states. It can be significantly shorter in nonstationary filters with time-varying parameters than in the structures with time-invariant parameters.

During previous studies on series-parallel structures the analysis of the stability of the time-varying parameters was conducted. It can be stated, that the process of settling parameters values over time is a strong factor stabilizing individual elements of the filter. Combining serial and parallel stable elements in one structure without any feedback allows to keep stability of the newly created structure. Stability tests conducted using Lyapunov's second method showed, that only fast, periodic changes of the filter parameters may result in instability. In analyzed filters such phenomenon does not occur.

For that reason, those basic dynamic nonstationary elements are worth to be examined and analyzed.

\section{Low Pass Elements with Time-Varying Parameters}

The simplest dynamic low-pass element with time-varying parameters is a structure described by the following differential equation:

$$
T_{\infty} \cdot f_{T}^{-1}(t) \cdot \frac{d y(t)}{d t}+y(t)=k_{\infty} \cdot f_{k}(t) \cdot x(t),
$$

for which we obtain easier implementation of the entire system.

Functions $f_{T}^{-1}(t)$ and $f_{k}(t)$ are described by dependencies

$$
\begin{gathered}
\left.f_{k}(t)=f_{k}(t) \cdot\left[d_{k}-\left(d_{k}-1\right) \cdot h_{k}(t)\right]\right) \\
f_{T}^{-1}(t)=f_{T}^{-1}(t) \cdot\left[d_{T}-\left(d_{T}-1\right) \cdot h_{T}(t)\right]
\end{gathered}
$$


where: $h_{k}(t)$ and $h_{T}(t)$ are step responses of second order elements with constant parameters.

Functions $\mathrm{f}_{\mathrm{T}}^{-1}(\mathrm{t})$ and $f_{k}(t)$ by varying the filter parameters allow to create gain function $k(t)=k_{\infty} \cdot f_{k}(t)$ and time constant function $T^{-1}(t)=T_{\infty}^{-1} \cdot f_{T}^{-1}(t)$, where $d_{T}=\frac{T^{-1}(0)}{T^{-1}(\infty)}$ and $d_{k}=\frac{k(0)}{k(\infty)}$ are the multiplicity of their value change. Gain function and time constant function must achieve the values specified in the spectrum assumptions in time not longer than the duration of the transient state. It can be assured if element generating varying function has damping factor value between $0,7 l<\beta<1$.

Because of the fact that each time at the beginning of filtration it is necessary to generate functions varying parameters, it was decided to use the step characteristics of second order oscillatory model with parameters $\mathrm{k}, \beta, \omega_{0}$ for this purpose. In spite of relatively simple implementation, it has a great capability of shaping desired functions.

Series connection of first order elements creates a filter, which order depends on number of elements. At this point the question arises: Could it be possible to unify all of the functions to one form and generate only one function without any significant properties deterioration in relation to optimal filters? The first results related to this topic were published in paper [6, 8]. Adopting these assumptions will reduce complexity of the structure without deteriorating properties in the time domain. That kind of filter, which is a connection of elements with the same time-varying functions, is described by following set of equations:

$$
\begin{gathered}
T_{\infty} \cdot f^{-1}(t) \cdot \frac{d y_{1}(t)}{d t}+y_{1}(t)=k_{\infty} \cdot f(t) \cdot x(t) \\
T_{\infty} \cdot f^{-1}(t) \cdot \frac{d y_{2}(t)}{d t}+y_{2}(t)=k_{\infty} \cdot f(t) \cdot y_{1}(t) \\
\vdots \\
T_{\infty} \cdot f^{-1}(t) \cdot \frac{d y_{i}(t)}{d t}+y_{i}(t)=k_{\infty} \cdot f(t) \cdot y_{i-1}(t) .
\end{gathered}
$$

The conducted study confirmed a very good properties of filters with the same variation of parameters. Examples of settling times with the input signal for step response and sinusoidal signal with admissible value $\alpha=0,05$ limiting the amplitudes of the frequency response are presented in Fig.1.

One can easily notice, that shortening the transient state of step response extends time of sinusoid attenuation and conversely. Furthermore, a solution can be found, where time of sinusoid attenuation and settling time for step input are equal. Fig.1 shows exact points of percolating planes presenting both settling times.

Fig.2 shows an example of random signal subjected to filtration. Fig. 3 presents an example filtration of the random signal with the use of first order filter with constant and variable parameters. Fig. 4 depicts filtration with the use of two first order filters connected in series (forming a second order filter).

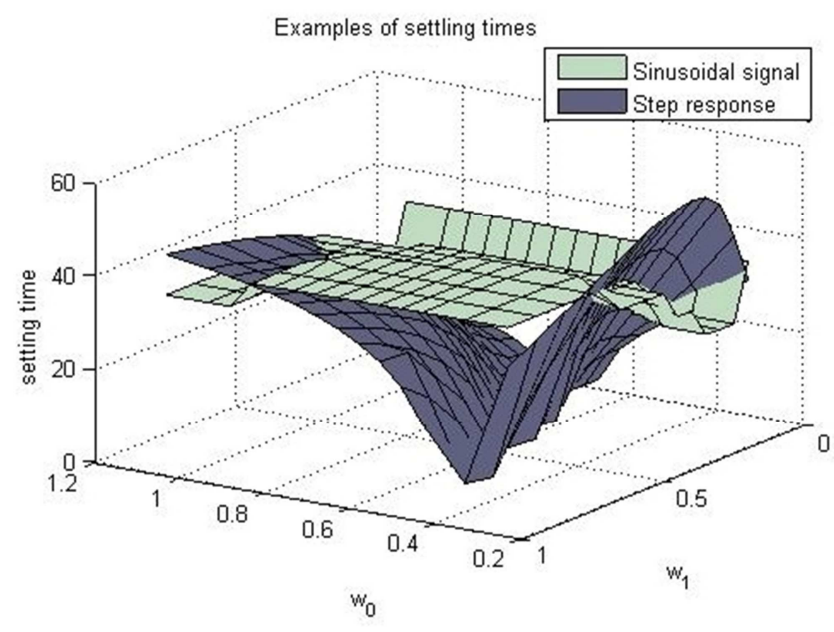

Figure 1. Examples of settling times for step response and sinusoidal signal for the first order filter $(\alpha=5 \%, d=3, T=20)$.

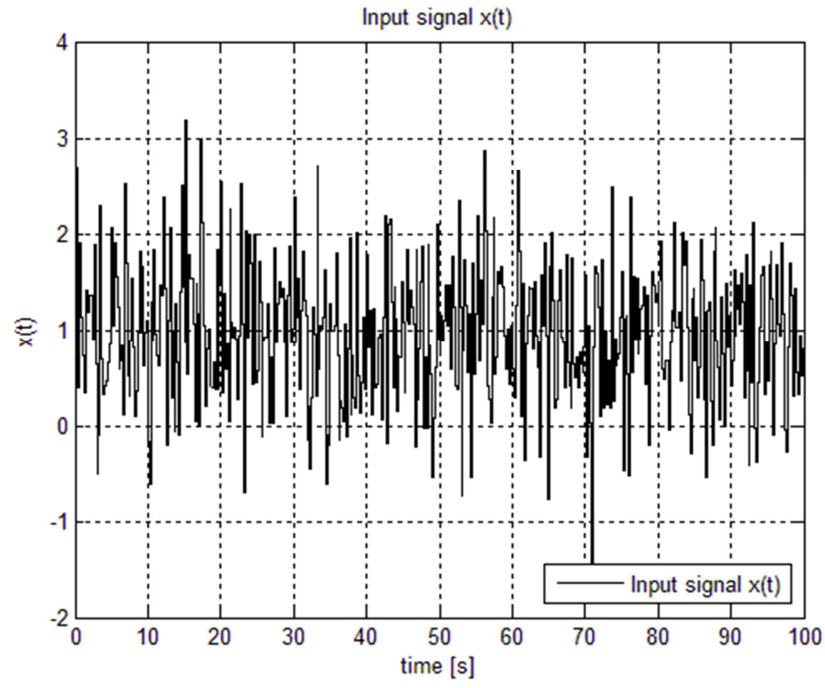

Figure 2. Random input signal.

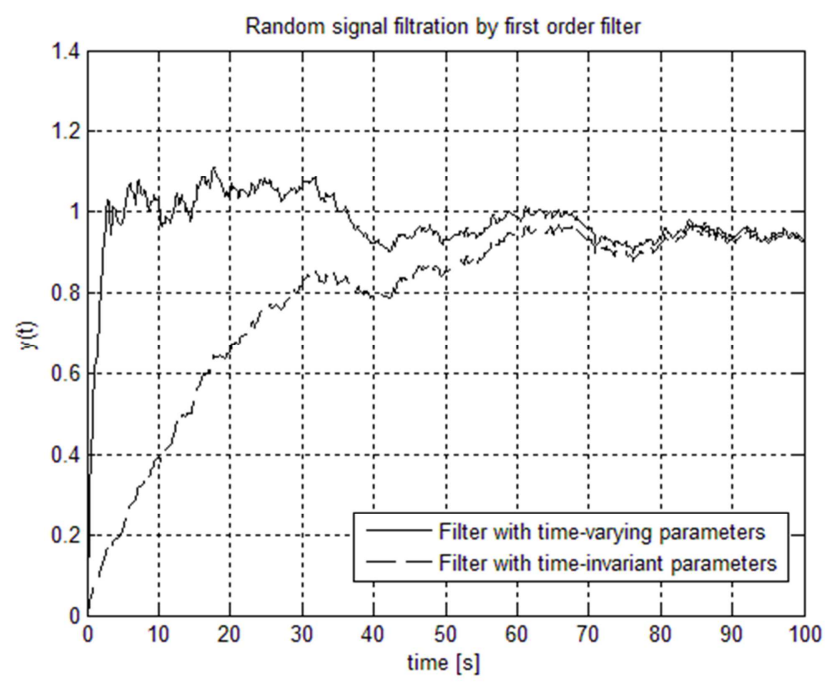

Figure 3. Random signal filtration by first order filter.

One can easily notice that with such selected parameters a single filter quite badly suppresses components with the 
lowest frequencies included in the filtered signal. Adding second equal element (Fig.4) provides an opportunity of better averaging the input signal. Simultaneously it can be seen, that filters with variable parameters have much shorter transient states compared to filters with constant parameters [3, 4]. Varying all parameters in all elements with one function allows to avoid making any mistakes related to elements connection order varied by different functions (in systems with time-varying parameters chain connection order has great importance in the duration of the transient state).

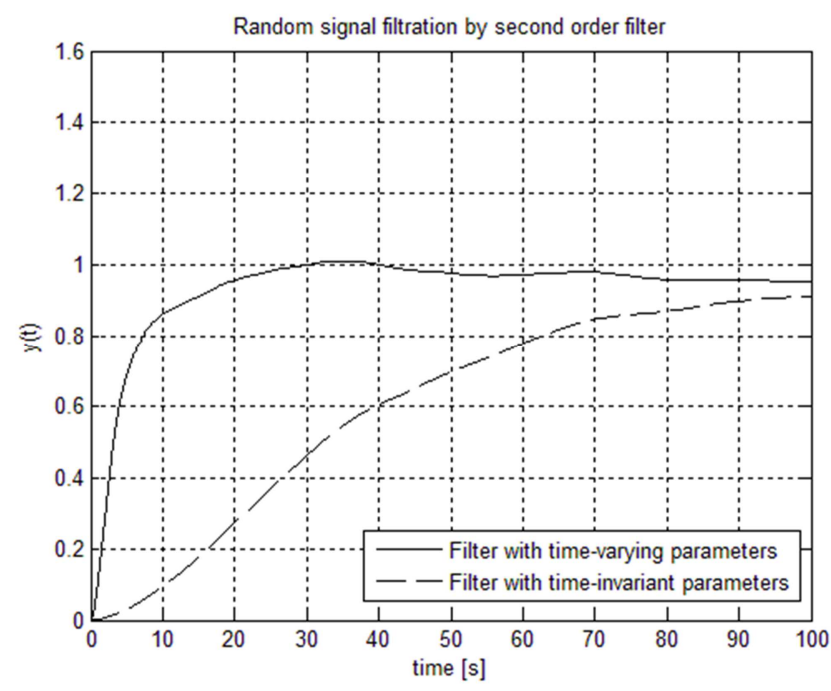

Figure 4. Random signal filtration by second order filter.

\section{High Pass Element with Time-Varying Parameters}

High-pass single element with time-varying parameters is described by the following equations:

$$
\begin{gathered}
T_{g_{n}} f_{T}^{-1}(t) \cdot \frac{d y(t)}{d t}+y(t)=k_{\infty} \cdot f_{k}(t) \cdot x(t) \\
z(t)=T_{g} \cdot \frac{d y(t)}{d t}
\end{gathered}
$$

where: $z(t)$ - high-pass element output signal, $T_{\mathrm{g}}-$ differentiation time.

Settling time of high-pass elements with time-varying parameters are not longer than the settling time of low-pass elements, from which they are built. The conclusion drawn from set of equations (3) is that the signal $z(t)$ at the output of high-pass element is proportional to the derivative of the signal $y(t)$ at the low-pass output of the same element with varying parameters. It can be assumed that the duration of the transient state depends of the lower limit of the high-pass band and can be phrased by the multiplicity of cutoff frequency period of the passband. Single high-pass elements with varying parameters can be a part of more complex structures. That would allow to create non-standard spectral characteristics of filters.

\section{Band Stop Element with Time-Varying Parameters}

Parallel connection of low-pass and high-pass elements depending on values of parameters can give diverse frequency characteristics. It can become phase shift corrector, band amplifying element or band-stop filter. This includes elements with time-invariant and time-varying parameters. Band-stop filter is obtained when low-pass element has lower limit frequency of the passband than the high-pass element. Elements with variable parameters provide a number of possibilities to accelerate the filtration.

Parallel connection of the low-pass and high-pass elements allows to obtain a signal, which is the sum of signals after low-pass and high-pass filtering

$$
u(t)=y_{d}(t)+z(t) .
$$

Fig.5 presents signal filtration, which is a sum of constant component and sinusoid with stopband range frequency.

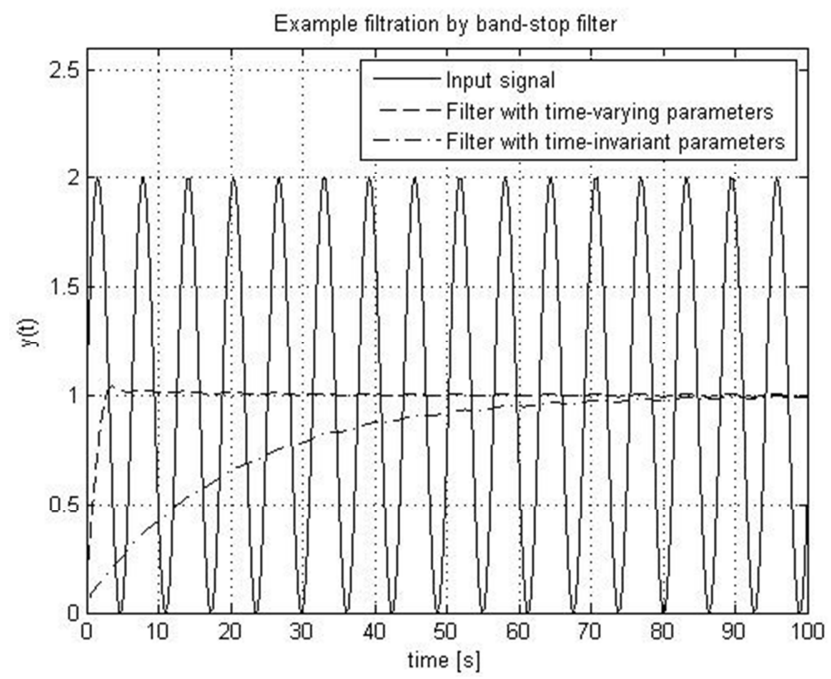

Figure 5. Signal filtration by band-stop filter.

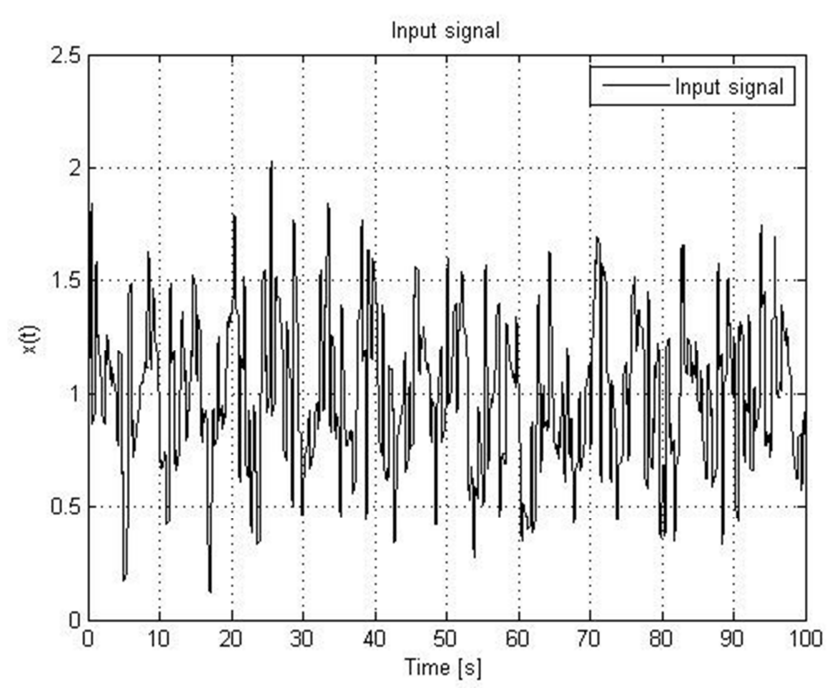

Figure 6. Random input signal. 


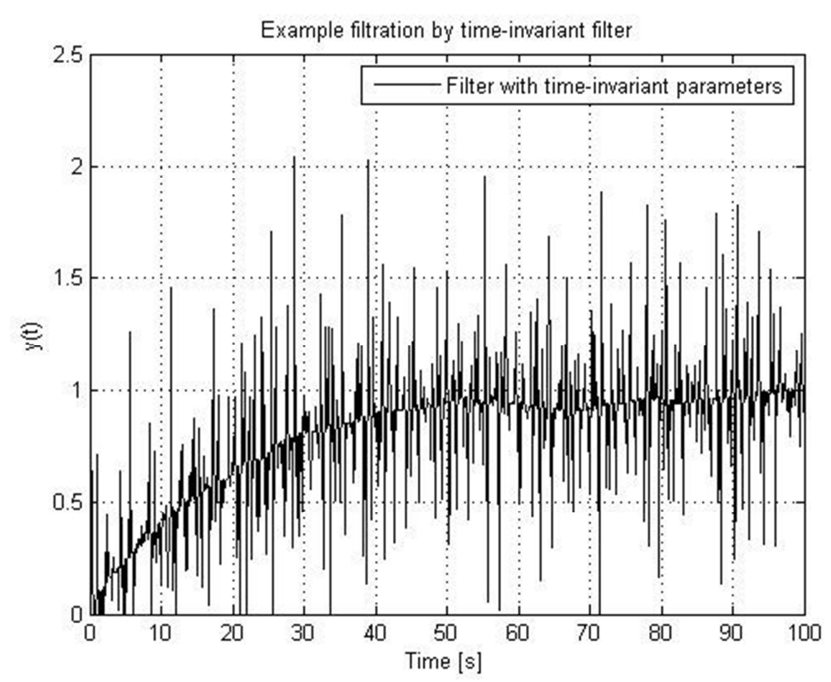

Figure 7. Filtration by filter with time-invariant parameters.

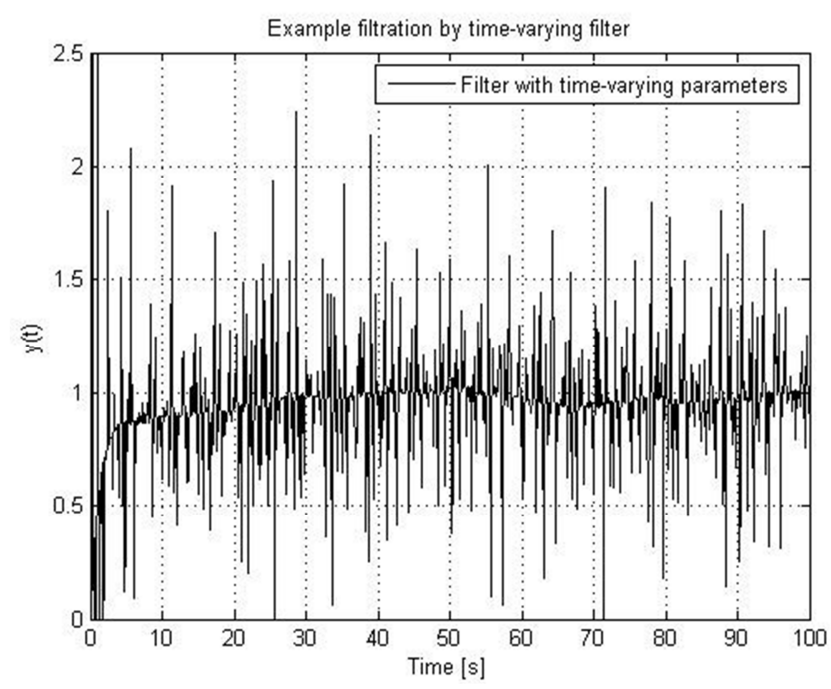

Figure 8. Filtration by filter with time-varying parameters.

As one can notice, the filter with time-varying parameters is characterized by much shorter transient state than the time-invariant filter. Based on the sample filtration of signal, which contains constant component, dominant sinusoid (e.g. derived from resonance phenomenon) and random noise, one can observe how the filtration with constant and varying parameters proceeds.

Fig.6 shows a signal subjected to filtration. Fig.7 presents filtration of that signal by using band-stop filter with fixed parameters. Fig. 8 depicts filtration by filter with time-varying parameters. Presented structure of filter is different from the notch type of structure, which is initially based on second order element, in which a zero amplitude for a single frequency can be reached. However, it is covered with a great oscillation - the greater, the bigger quality factor is. In the following examples it is clear, that filter manages to suppress the sinusoid component and the transmission of other components.

Connecting in series similar pairs of elements creates a parallel-serial structure. It allows to obtain the frequency characteristics with more narrow slopes as shown in Fig.9.

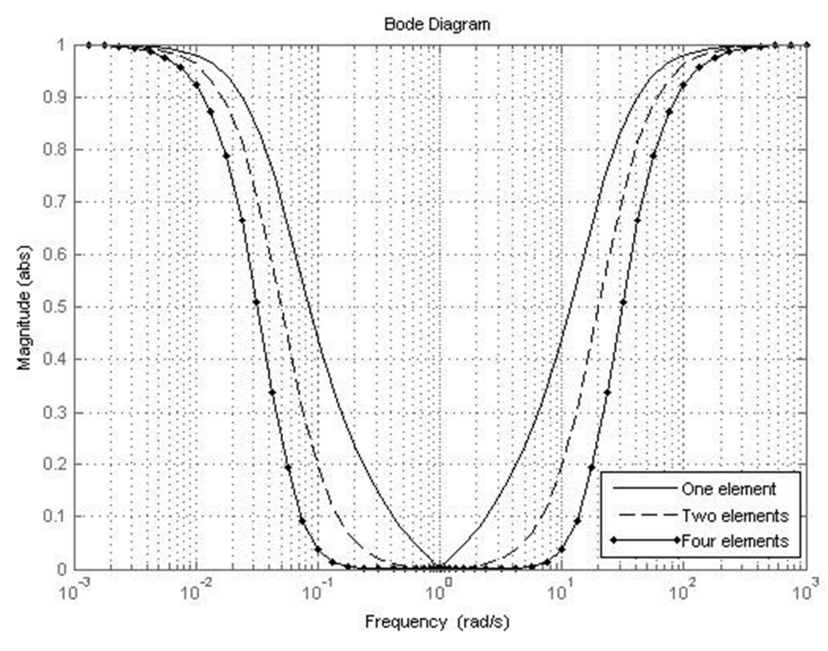

Figure 9. The amplitude characteristics of series connected filters.

Presented basic elements with time-varying parameters can be arranged in more complex structures. Each of low-pass element contains in its structure a high-pass output. One of the main disadvantage of those simplest elements with constant parameters are very long transient states. By introducing the time-varying parameters to those structures, the transient states can be reduced. They are characterized by very good properties in the time domain. Spectral characteristics can be improved and arbitrarily formed through the expansion of the structure. For example, the combination of two or more band-stop components with different values of stopband range frequency can be used in filtering signals with many resonant frequencies generated in circuits. Parallel-series structures in order to achieve non-standard spectral characteristics while maintaining good dynamic properties of these structures can be created. By using single nonstationary elements one can create structures providing even more demanding frequency characteristics. At the same time they do not suffer from many drawbacks, which filters with constant parameters are characterized with. Created structures shown in Fig.10 (or similar), that have a layered or any other parallel-series structure can be subjected to learn required properties.

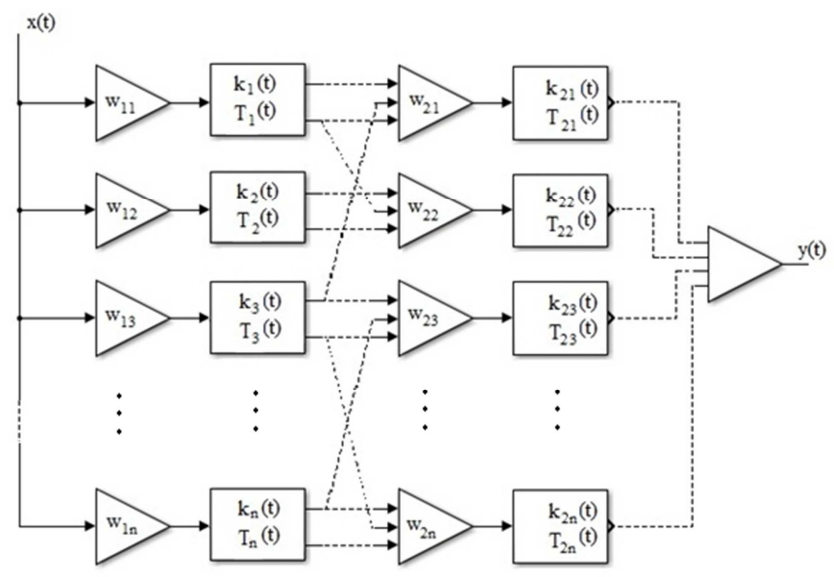

Figure 10. Example of parallel-series structure. 


\section{Conclusions}

Conducted study has shown, that the same functions varying different parameters of the time-varying structures may significantly simplify the whole design and can provide reduced transient state time. Such simplification of the individual elements allows to combine many of them, creating expanded filter structures with unique spectral characteristics. Presented examples of filtration provide a comparison of efficiency between time varying and time-invariant filters with the same spectral properties. Creating a parallel-serial structure gives a possibility to form various frequency characteristics. Obtained structures are characterized by very high stability resulting from the simplest basic elements and the lack of feedback inside the whole system structure.

\section{References}

[1] Anjum S.R., Bhattacharya S., Srirastava G., Effect of Phase Compensation on the performance of Classic Butterworth filter, Proc.ICAESM, pp.217-221, 2012.

[2] Carusone A., Johns D.A., Analogue adaptive filters: past and present. IEE Proc.-Circuits Devices Systems, vol. 147, No. 1, pp. 82-90, Feb. 2000.

[3] Chang-Siu E., Tomizuka M., and Kong K., Time-Varying Complementary Filtering for Attitude Estimation, Proc. IEEE/RSJ, pp. 2474-2480, 2011.

[4] Chen W.K., The Circuits and Filters Handbook, CRC Press, IEEE Press, Boca Raton, 2003.
[5] Gutierrez de Anda M.A., Sarmiento Reyes L.A., The analytic determination of the PPV for second-order oscillators via time-varying eigenvalues, IEEE Transactions on Cirtuits and Systems II: Express Briefs, vol. 53, (2006), no. 11, pp.1225-1229.

[6] Gutierrez de Anda M.A., Sarmiento Reyes L.A., Martinez L.H., Piskorowski J. and Kaszyński R., The reduction of the duration of the transient response in a class of continuous-time LTV filters, IEEE Transsactions on Circuits and Systems-II: Express Briefs, vol. 56, (2009), no. 2, pp. 102-106.

[7] Jafaripanah M., Al.-Hashimi B.M., White N.M., Application of analog adaptive filters for dynamic sensor compensation, IEEE Transactions on Instrumentation and Measurement, vol. 54, No. 1, pp. 245-251, Feb. 2005.

[8] Lee H., Bien Z., Linear time-varying filter with variable bandwidth, in Proc. IEEE International Symphosium on Circuits and Systems ISCAS (2006), pp. 2493-2496.

[9] Piskorowski J., Kaszynski R. Analytical synthesis of parameter-varying filter of constant component with application to swiching systems, Metrology and Measurement Systems, vol. XVIII (2011) no. 3, pp. 471-480.

[10] Piskorowski J., Phase-Compensated Time-Varying Butterworth Filter, Analog Integrated Circuits and Signal Processing, Springer US, 2013.

[11] Toledo K., Torres J. A Variational Approach for Designing Digital Filters with Time-Varying Parameters, IEEE Transsactionson Circuits and Systems II: Express Brieffs, vol. X No. X, Jan. 2015.

[12] Walczak J., Piwowar A., Cascade connection of parametric sections and its properties, Electrical Review, Vol. 86, No. 1, pp. 56-58, Jan. 2010. 